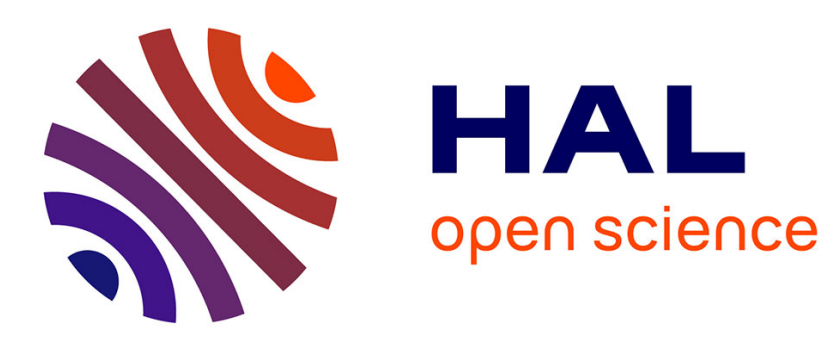

\title{
Physical parameters of the violin bridge changed by active control
}

Henri Boutin, Charles Besnainou

\section{To cite this version:}

Henri Boutin, Charles Besnainou. Physical parameters of the violin bridge changed by active control. Acoustics'08, Jun 2008, Paris, France. hal-02470042

\section{HAL Id: hal-02470042 \\ https://hal.science/hal-02470042}

Submitted on 7 Feb 2020

HAL is a multi-disciplinary open access archive for the deposit and dissemination of scientific research documents, whether they are published or not. The documents may come from teaching and research institutions in France or abroad, or from public or private research centers.
L'archive ouverte pluridisciplinaire HAL, est destinée au dépôt et à la diffusion de documents scientifiques de niveau recherche, publiés ou non, émanant des établissements d'enseignement et de recherche français ou étrangers, des laboratoires publics ou privés. 

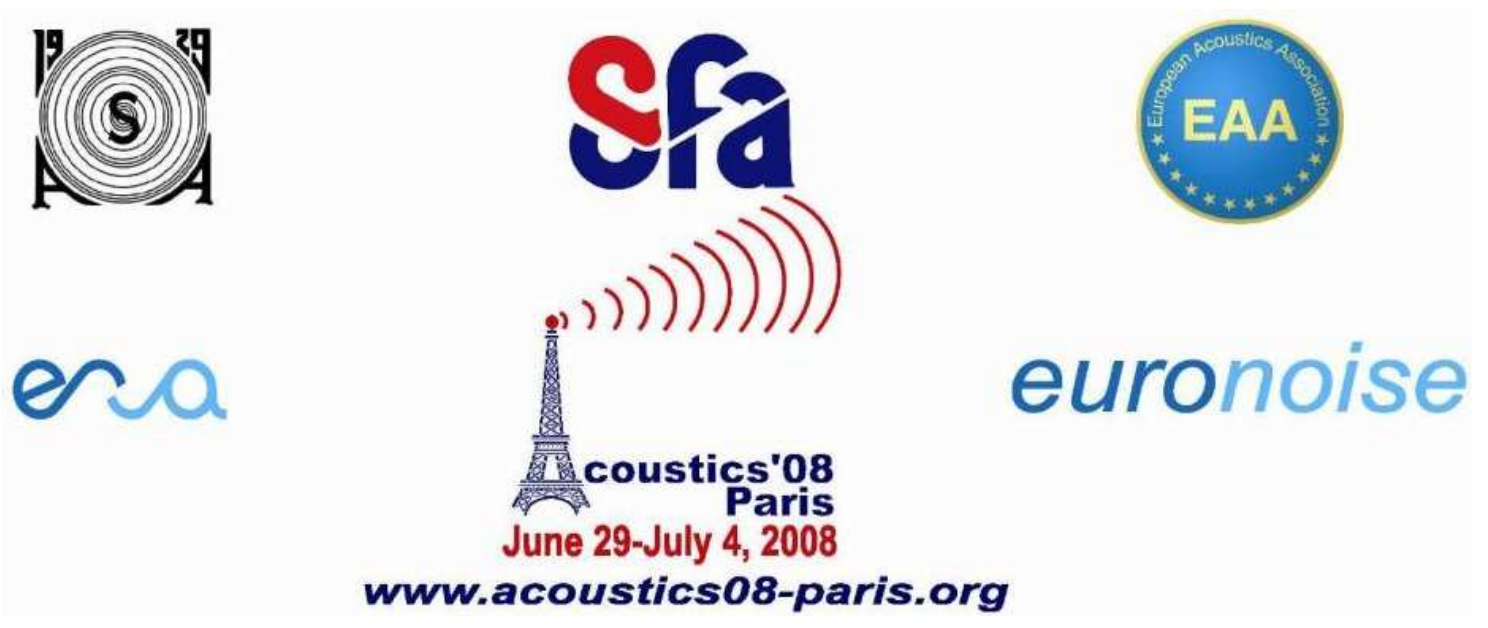

\title{
Physical parameters of the violin bridge changed by
} active control

\author{
H. Boutin ${ }^{a}$ and C. Besnainou ${ }^{b}$
}

anstitut Jean le Rond d'Alembert, Lab. d'Acoustique Musicale, 11, rue de Lourmel, 75015 Paris, France

bInstitut Jean le Rond d'Alembert, Laboratoire d'Acoustique Musicale, 11, rue de Lourmel, 75015 Paris, France

boutin@lam.jussieu.fr 
On the input admittance of many violins a typical broad frequency peak, called "bridge hill" appears around $2.5 \mathrm{kHz}$. The physical parameters of a violin bridge have a significant influence on this feature, and then on the tonal colouration of the produced sound.

The effect of the bridge characteristics (mass, stiffness and foot spacing) on the frequency response have been revealed by using bridge models through several studies.

Here the parameters of the bridge response are changed, by applying an active control method. Such a correction, very useful in noise reduction, enables to change separately the position and the shape of the bridge hill. It is first tested on a simplified bridge model, then on a real bridge by placing 2 actuators and an accelerometer at strategic positions.

\section{Introduction}

The sound production of most bowed string instruments is due to three essential components: the strings which are excited by the musician, the instrument body which radiates sound all around, thanks to the movement of the top and back plates, and the bridge, Fig.1, which transmits the vibrations between the string and the body. Its response has a determining influence on the resulting sound.

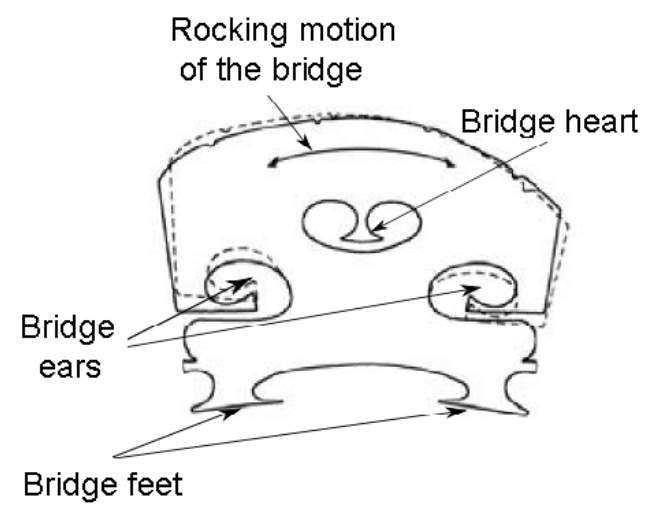

Figure 1: Example of a violin bridge.

The instrument makers are aware of its role and attach importance to its adjustement. Several frequency response measurements of high quality violins revealed the existence of a broad peak between 2 and $3 \mathrm{kHz}$, followed by a steady drop in amplitude at higher frequencies[1]; an example is plotted in Fig.2. This feature is called "bridge hill" because first, Cremer attributed its existence to the lowest resonance of the bridge [2].
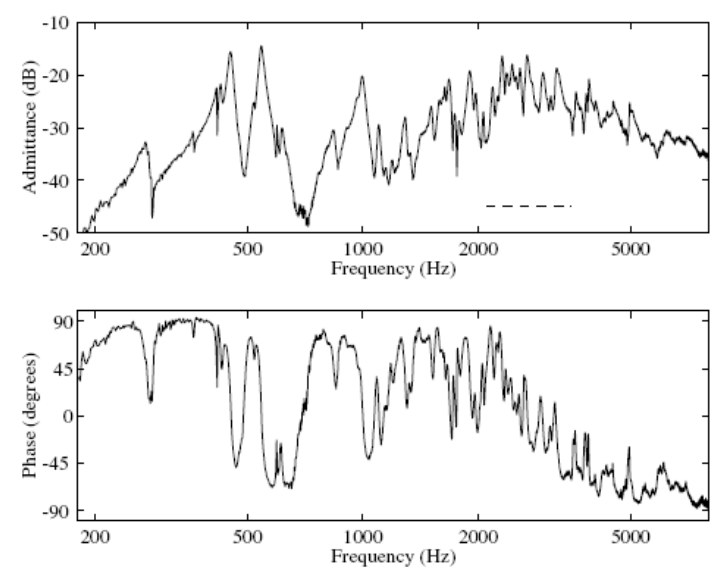

Figure 2: Input admittance of a real violin bridge.
Then many studies have proposed bridge models in order to explain the cause of this hill and to make clear the effect of the bridge physical parameters on it. By analysing Reinicke's mass-spring model, Beldie showed that the hill center frequency depends on its stiffness $[3,4]$. Then Woodhouse carried out a simplified model, Fig.3, and studied the influence of its mass and of its stiffness on the "bridge hill" shape [5].

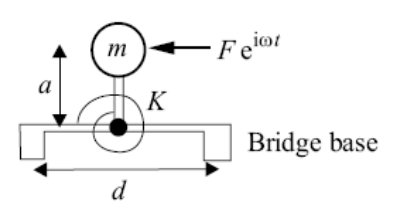

Figure 3: The simplified bridge model proposed by Woodhouse.

This paper proposes an other idealised bridge model in Fig.4, simple enough to foresee its behaviour. First it is described and compared to the models introduced above. Then its physical parameters are changed in order to find out their effect on its frequency response. In the last part an additional couple of forces is exerted on the model to modify the bridge hill shape in real time.

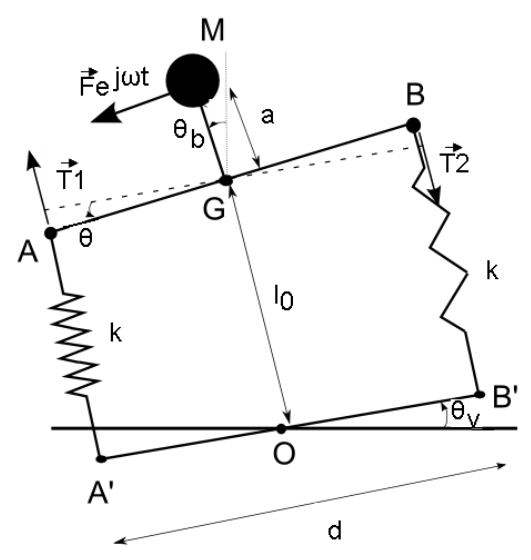

Figure 4: An other violin bridge model.

\section{An other violin bridge model}

The purpose of this study is to change the bridge response of a violin with an active control method. After applying this feedback correction on a bridge model, it is intended to be tested on a real bridge by using one sensor and two actuators. Their localisations are all the 
easier to find since the model looks real. Because of this last issue the torsion spring of Woodhouse's model is replaced here by two classic springs of stiffness $k$, which can only move in phase opposition. Thus they enable the bridge to get a rotational movement. As in Woodhouse's model, only the upper part mass is not neglected. It is divided in two components, cf Fig.4: the point $M$, where the strings rest $($ mass $=m 1)$, and the horizontal piece linked to the springs at points $A$ and $B$ (mass $=m_{2}$, length $=d) . a$ is the length of the rotating link between $M$ and $G$, and $l_{0}$ is the length of the springs. $\theta$ is the angle between $[A B]$ and the bridge base, whereas $\theta_{v}$ is the angle between the bridge base and the horizontal rest position of the violin plate. Their sum is called $\theta_{b}$. The upper part of the bridge is subjected to the force applied by the string in $M, F e^{j \omega t}$, and the forces applied by the springs in $A$ and $B: T 1$ in $A$ and $T 2$ in $B$. The sum of their moments, calculated around the fixed point $O$ is :

$$
F e^{j \omega t}\left(l_{0} \cos (\theta)+a\right)-\sin (2 \theta) \frac{d^{2} k}{4}=J j \omega \frac{V_{b}}{l_{0}+a}
$$

where $J\left(=m_{1} a^{2}+\frac{m_{2} d^{2}}{12}\right)$ is the moment of inertia of the bridge upper part and $V_{b}$ is the linear velocity of the point $M$. The bridge admittance $Y_{b}$, defined as the quotient $\frac{\theta+\theta_{v}}{F e^{j \omega t}}$ is obtained by differentiating the equation (1). Assuming that $\theta$ is a very small angle, $\cos (2 \theta) \approx$ $\cos (\theta) \approx 1$ and $\sin (\theta)<<\cos (\theta)$; hence

$$
Y_{b}=\left(l_{0}+a\right)^{2} \frac{j \omega+\frac{k d^{2} R}{2}}{(j \omega)^{2} J+\frac{k d^{2}}{2}(1+J R j \omega)}
$$

Woodhouse [5] led an equivalent calculation by using Reinicke's model with a torsion spring $k^{\prime}$, a punctual mass $m^{\prime}$ and a rotating link length $a^{\prime}$. He gets the same results with $k^{\prime}=k d^{2} / 2, m^{\prime}=J /\left(l_{0}+a\right)^{2}$ and $a^{\prime}=l_{0}+$ a. As its resonance frequency is $\frac{1}{a^{\prime}} \sqrt{\frac{k^{\prime}}{m^{\prime}}}$, in our model it is defined by $\Omega_{b}=d \sqrt{\frac{k}{2 J}}$. Therefore $k=2 J\left(\frac{\Omega_{b}}{d}\right)^{2}$.

The rotational admittance is expressed by using the violin model of Woodhouse [5]. It incorporates the top and back plates of the body as two rectangular bending plates with hinged boundary conditions all around. It also includes the soundpost as a massless and rigid link between both plates. The velocity of the plates at the contact points is the same. $R$ is plotted in Fig.5. With such a model, it is composed by a set of eigen modes.

The constants $l_{0}, a, d$ and $J$ that appear in the expression of $Y_{b}$ are estimated with a real violin bridge:

$$
\begin{aligned}
& -l_{0}=0.01 \mathrm{~m} \\
& -a=0.03 \mathrm{~m} \\
& \text { - } d=0.03 \mathrm{~m} \\
& \text { - } m_{t o t}=0.5 \mathrm{~g} \text { and } m_{1}=m_{2}=m_{t o t} / 2 \\
& \text { - } J=m_{1} a^{2}+\frac{m_{2} d^{2}}{12}=2.4375 \cdot 10^{-7} \mathrm{~kg} \cdot \mathrm{m}^{2}
\end{aligned}
$$

On the measurement of bridge mobility carried out by Jansson and Bissinger $[6,7]$, no isolated bridge resonances were observed. Here the chosen value of $\Omega_{b}$ is $2 \pi \times 3 \mathrm{kHz}$, so that it approximatively fits in with the lowest bridge in-plane resonance. Consequently the stiffness of each spring is $k=192457 N . m^{-1}$. On Fig.6, $Y_{b}$
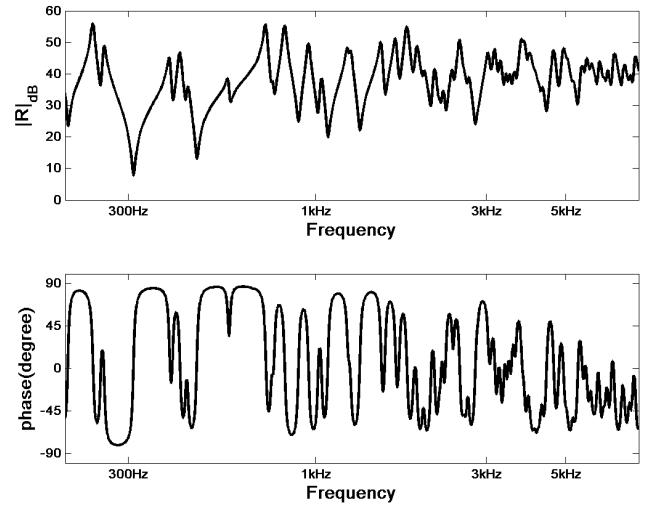

Figure 5: Rotational admittance calculated with Woodhouse's violin model.

is plotted and the bridge hill is guessed around $2.8 \mathrm{kHz}$. Thus the described model with two springs is in accordance with the earlier models using a torsion spring.
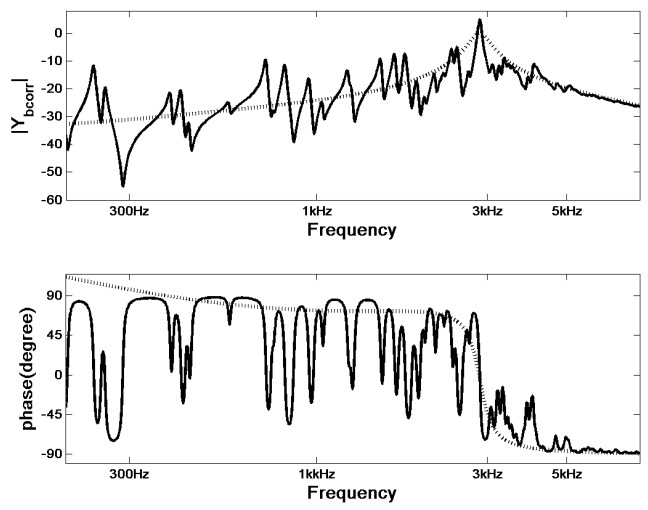

Figure 6: Bridge mobility calculated with the bridge model described here

\section{Changing the bridge physical parameters}

The previous part was dedicated to describe a bridge model and to plot its frequency response. Now it would be interesting to assess the influence of its physical parameters on it. It is therefore essential to display the bridge hill without the resonance peaks of the rotationnal admittance, in order to measure its characteristics accurately.

\subsection{How to measure the bridge hill char- acteristics}

Woodhouse explains why those peaks disappear if the violin top and back plates are replaced by infinite plates [5]. Actually no wave propagating on such a body is reflected, because its boundaries are infinitely far away. Thus no resonance peak occurs, since no standing wave settles. 
If the violin body model is modified, then the expression of $R$ also changes. Actually it depends on the violin body admittance at the bridge feet and at the soundpost position [5], and now those magnitudes are different since the violin plates are infinitely large. The new rotationnal admittance is called $R_{\infty}$. The bridge admittance is calculated again, $Y_{b \infty}$ while replacing $R$ by $R_{\infty}$ in $(2)$ :

$$
Y_{b \infty}=\left(l_{0}+a\right)^{2} \frac{j \omega+\frac{k d^{2} R_{\infty}}{2}}{(j \omega)^{2} J+\frac{k d^{2}}{2}\left(1+J R_{\infty} j \omega\right)}
$$

It is plotted in dashed line on Fig.6. The resonance peaks due to the rotationnal admittance have been removed. Such a smooth bridge response is called a skeleton curve by Woodhouse [5]. Now the bridge appears clearly and looks like the frequency response of a second order filter. Its eigen frequency, its damping factor and its maximum gain can be easily measured and compared to those of other bridge responses.

\subsection{Influence of some bridge physical pa- rameters on the bridge hill}

The next stage consists in discussing the effect of some physical bridge characteristics on the width, the height and the place of the hill. While a violin maker can not easily change the geometric characteristics of the bridge without modifying the playing comfort, he would rather control independently the bridge mass and the stiffness. The total mass can be reduced by removing matter from the "bridge heart", whereas the stiffness can decrease by digging the "bridge ears", cf Fig.1. The result of these alterations is looked in the bridge model by varying the values of the mass and of the stiffness in the expression of $Y_{b \infty}$ :
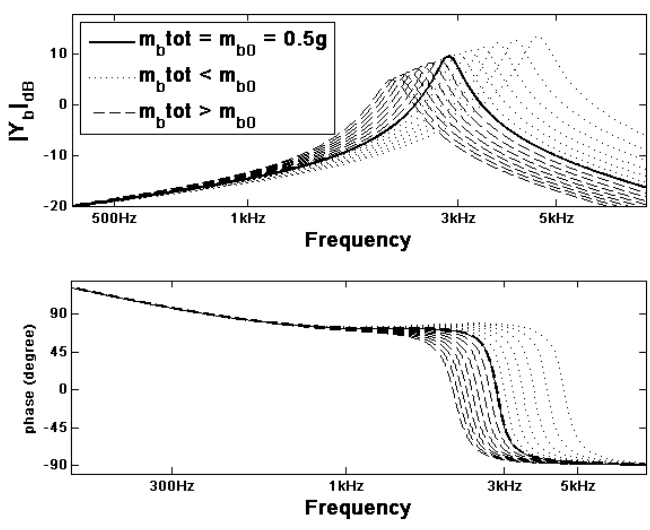

Figure 7: Variation of the bridge's mass

On the Fig.7, the total mass $m_{\text {total }}$ is increased while keeping $k$ fixed. In this case, the bridge hill center frequency, the damping factor and the maximum gain decreases.

On the Fig.8, the stiffness $k$ is increased while keeping $m$ fixed. Hence the hill frequency gets higher whereas the damping factor and the maximum gain decreases.
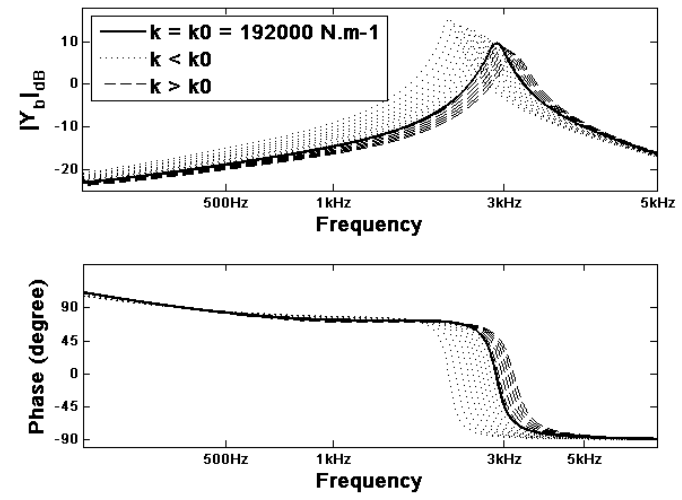

Figure 8: Variation of the bridge's stiffness

These results confirm those achieved by Woodhouse [5] and Beldie [4]. They are also in accordance with the behaviour of a simple $2^{\text {nd }}$ order harmonic oscillator mass $M$, stiffness $K$, damping $R$ which resonance frequency and damping factor are respectively $\sqrt{\frac{K}{M}}$, and $\sqrt{\frac{K M}{R}}$.

\section{Correction of the violin sound parameters by active control}

The bridge filters the excitation provided by the strings vibrations. Thus, while changing its frequency response, the instrument timbre gets modified.

\subsection{Definition of the parameters to change in the violin response}

Some harmonics of the played notes can be emphasized or dampened by changing the position of the bridge response bandwidth, its amplitude and its selectivity. Consequently controlling the resonance frequency, the damping factor and the maximum gain of the bridge admittance should enable to modify the violin's sound identity.

The expression of $Y_{b \infty}$ is very similar to the frequency response of a $2^{\text {nd }}$ order harmonic oscillator. Indeed, by using Eq.(3), its eigen frequency, damping factor and maximum gain are respectively defined as:

$$
\begin{aligned}
f_{0 b} & =d \sqrt{\frac{k}{2 J}}=d \sqrt{\frac{k}{2\left(m_{1} a^{2}+\frac{m_{2} d^{2}}{12}\right)}} \\
Q_{b} & =\frac{1}{d R \sqrt{\frac{k J}{2}}}=\frac{\sqrt{(2)}}{d R \sqrt{k\left(m_{1} a^{2}+\frac{m_{2} d^{2}}{12}\right)}} \\
H_{0 b} & =\frac{\left(l_{0}+a\right)^{2}}{J}\left(\frac{1}{j \omega}+\frac{2}{k R d^{2}}\right)
\end{aligned}
$$

Since the moment of inertia $J$ and the total mass $m_{\text {total }}$ vary in the same way, the phenomena observed on the skeleton curves (figures $\underline{[8]}$ and [9]) become clear: $Q_{b}$ and $H_{0 b}$ are all the more decreased when $J$ or $k$ are increased, Eq.(5) and (6), and $f_{0 b}$ gets higher when $k$ increases and lower when $m$ increases, Eq.(4). 


\subsection{Correction by active control}

Changing the bridge mass and stiffness causes simultaneous modifications of the height, the width and the place of the hill. A PID correction applied to a $2^{\text {nd }}$ order harmonic oscillator enables to bring its resonance frequency, its Q-factor and its maximum gain under independant control.

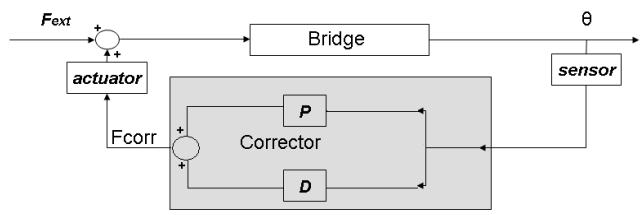

Figure 9: Feedback correction applied on a real bridge

In such a looped system, plotted in Fig.9, $u$ is the oscillation velocity. The feedback force generated by the PID corrector is $f_{\text {corr }}=P u+I \frac{u}{j \omega}+D j \omega u$, so that the controlled oscillator gets new parameters $f_{0}^{\prime}, Q^{\prime}$ and $H_{0}^{\prime}$. In this case the coefficients $P, I$ and $D$ can be expressed in function of their relative variations $\Delta \omega_{0}, \Delta Q$ and $\Delta H_{0}$ [8].

However the described bridge model placed on an infinitely large body is not really a $2^{\text {nd }}$ order harmonic oscillator. Indeed its parameters $f_{0}, Q$ and $H_{0}$ are not constant since the rotational admittance depends on the frequency Eq.(4),(5) and (6). Despite that, let us focus on the effect of a PID correction on the bridge hill parameters. A couple of forces are applied on the bridge upper part, so that their resulting moment is $P \dot{\theta}_{b}+I \theta_{b}+D \ddot{\theta}_{b}$, cf Fig.4. Because of this additionnal moment, the bridge mobility is modified:

$$
Y_{b \infty}=\frac{\left(l_{0}+a\right)^{2}\left(j \omega+k^{\prime} R_{\infty}\right)}{(j \omega)^{2} \alpha+j \omega \beta+\gamma-\frac{I \delta}{j \omega}}
$$

where $k^{\prime}=\frac{k d^{2}}{2}$,

$\alpha=(J-D)$,

$\beta=(J-D) R_{\infty} k^{\prime}-P$,

$\gamma=-I+k^{\prime}\left(1-R_{\infty} P\right)$,

and $\delta=k^{\prime} R_{\infty}$.

In Eq.(7), the denominator remains a $2 n d$ degree polynomial only if $I=0$. In this case, the new parameters $\omega_{0}^{\prime}, Q^{\prime}$ and $H_{0}^{\prime}$ depend on $\mathrm{P}$ and $\mathrm{D}$ :

$$
\begin{aligned}
\omega_{0}^{\prime} & =\sqrt{\frac{k^{\prime}\left(1-R_{\infty} P\right)}{J-D}} \\
Q^{\prime} & =\frac{\sqrt{k^{\prime}\left(1-R_{\infty} P\right)(J-D)}}{(J-D) k^{\prime} R_{\infty}-P} \\
H_{0}^{\prime} & =\frac{\left(l_{0}+a\right)^{2}}{(J-D) k^{\prime} R_{\infty}-P}\left(1-\frac{j k^{\prime} R}{\omega}\right)
\end{aligned}
$$

This equations system gives expressions of $\mathrm{P}$ and $\mathrm{D}$ in function of the input values $\Delta \omega_{0}$ and $\Delta Q$ :

$$
P=\frac{1}{\frac{(1+\Delta Q)\left(1+\Delta \omega_{0}\right)^{2}}{R_{\infty} k^{\prime} J\left(\Delta Q-\Delta \omega_{0}\right)}+R_{\infty}}
$$

$$
D=J\left(1-\frac{1+\Delta Q}{(1+\Delta Q)\left(1+\Delta \omega_{0}\right)^{2}+R_{\infty}^{2} k^{\prime} J\left(\Delta Q-\Delta \omega_{0}\right)}\right)
$$

An other relation is given by the system, between $\Delta H_{0}$, and the coefficients $P$ and $D$ :

$$
\Delta H_{0}=\frac{1}{\frac{J}{D+\frac{P}{k^{\prime} R_{\infty}}}-1}
$$

Therefore $\Delta H_{0}$ can be deduced from $\Delta Q$ and $\Delta \omega_{0}$. As a conclusion, while $I=0$ the feedback loop imposes the value of two parameters, $Q$ and $\omega_{0}$ for instance, without controlling the third variation $\Delta H_{0}$. The Fig.11 and Fig.12 display different bridge responses resulting of some $P D$ corrections:
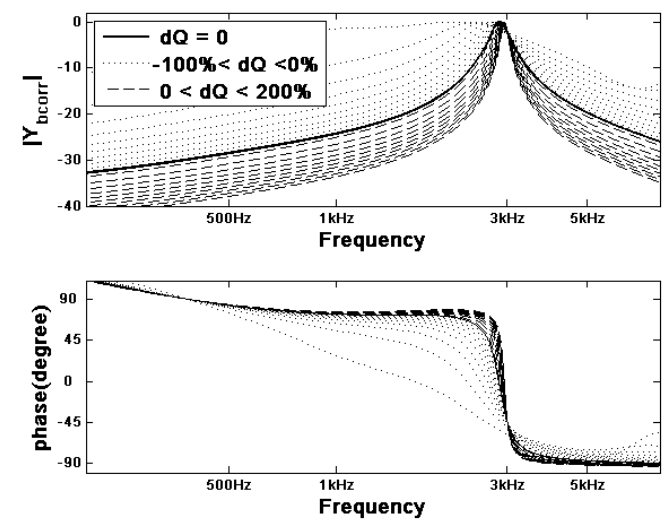

Figure 10: Correction of the damping factor with an "ideal" PD corrector
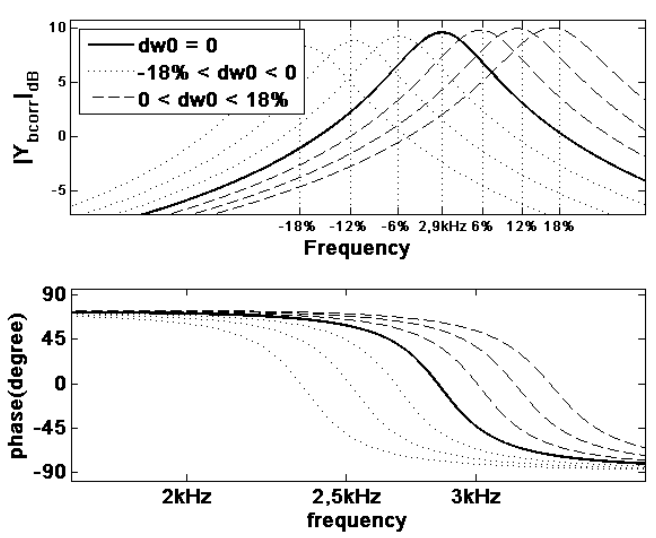

Figure 11: Correction of the resonance frequency with an "ideal" PD corrector

First, on Fig.10, the damping factor is varied while imposing $\Delta \omega_{0}=0$. The skeleton curves have been normalized. In this way, $\Delta H_{0}$ can not appear and the $\mathrm{Q}$ variations are clearly perceived. All the same, the resonance frequency of the bridge hill changes although it should remain constant. Especially, $\omega_{0}$ decreases while $\Delta Q<0$. Then, on Fig.11, $\omega_{0}$ is modified while keeping $\Delta Q=0$. Here the curves are not normalized. That is why some gain modifications appear. The Q-factor seems to remain constant. However the $\omega_{0}$ variations do not achieve the exact desired correction. In both extreme cases $\Delta \omega_{0}>-18 \%$ and $\Delta \omega_{0}<18 \%$. 
Actually as $R_{\infty}$ is a complex value, $\omega_{0}, Q$ and $H_{0}$ are also complex. Therefore their value are not the resonance frequency, damping factor and maximum gain that are actually measured on the figure, because their imaginary part is not equal to zero. That is why the hill is not exactly centered in $3 \mathrm{kHz}$. Moreover $\omega_{0}, Q$ and $H_{0}$ depend on $R_{\infty}$ and then also depend on $\omega_{0}$. Consequently, as the $\mathrm{P}$ and $\mathrm{D}$ coefficients of the controller are deduced from those parameters, they are not constant values either. To carry out a simplified correction on a real bridge, $R_{\infty}$ just needs to be replaced by a constant value in the expression of $P$ and $D$.

\subsection{How to apply the correction on a real bridge}

This last paragraph proposes a method for controlling a real violin's bridge response. The $P D$ correction described above is obtained by applying an additionnal couple of forces, which moment depends on the bridge's angular velocity. This force signal can be generated by using an accelerometer that measures $\theta_{b}$. Then the controller calculates the $P$ and $D$ coefficients in function of the $\Delta \omega_{0}$ and $\Delta Q$ variation desired by the user in real time.It deduces the value of the following force signal: $F_{c o r r}=\frac{1}{d}\left(P \dot{\theta}_{b}+D \ddot{\theta}_{b} u_{r}\right)$ Two actuators have been wedged in the "bridge ears" to apply this correction to the mobile part of the bridge. The system is described in Fig.12.

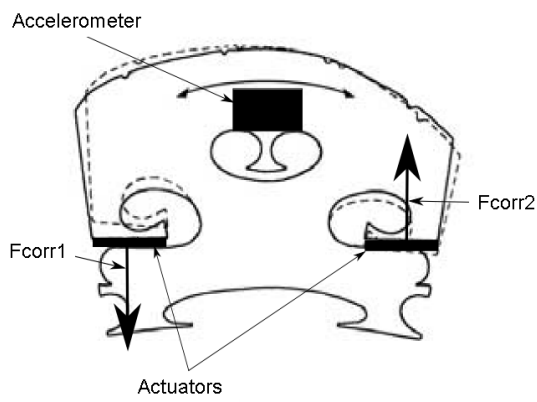

Figure 12: Real bridge provided with 2 actuators and 1 accelerometer

Actually $F_{\text {corr }}$ is send to the first actuator (for instance placed in $A$ ), and its opposite to the other one (placed in $B$ ). Assuming that the actions of both actuators are perpendicular to the bridge base, then the resulting moment around $O$ is:

$$
M_{F_{\text {corr }}}=P \dot{\theta}_{b}+D \ddot{\theta}_{b}
$$

Thus, the movement of the real bridge should be described by Eq.(7). Consequently its frequency response must get modified as observed in Fig.10 and Fig.11, according to the correction imposed by the instrumentist. been used here to change the frequency behaviour of a violin bridge. In particular the purpose was to control the shape of the "bridge hill", since its influence on the violin sound is significant. Thanks to a feedback correction, the resonance frequency and the damping factor of the bridge response have been brought under independent control. Those results have been obtained by controlling a very simplified bridge model. Such a correction has also been carried out on a real bridge and has permitted to hear timbre modifications in the produced violin's sound.

\section{Acknowledgments}

A lot of thanks to Nicolas Pailheret that has actively participated to this study.

\section{References}

[1] L. Cremer, Physik der Geige (The physics of the violin), Wissenschaft der VG WORT GmbH, München, Universität Berlin, S. Hirzel Verlag Teil II Kap. 1 "Der Steg", 171-180 (1981)

[2] E.V. Jansson, "Admittance measurements of 25 high quality violins", Acta Acustica united with Acustica 83, 337-341 (1997)

[3] W. Reinicke, "Die Übertragungseigenschaften des Streichinstrumentensteges" (Transfer characteristics of string instrument bridges), Thesis, Technische Universität, Berlin (1973)

[4] I.P. Beldie, "About the bridge hill mistery", J. Catgut Acoust. Soc. Series 2 4, 9-13, (2003)

[5] J. Woodhouse, "On the "bridge hill" of the violin", Acta Acustica united with Acustica 91, 155-165 (2005)

[6] E.V.Jansson, "Violin frequency response - bridge mobility and bridge feet distance", Applied Acoustics 65, 1197-1205 (2004)

[7] G. Bissinger, "The violin bridge as filter" J. Acoust. Soc. Am. 120, 482-491 (2006)

[8] H. Boutin, "Contrôle actif sur instruments acoustiques", Master ATIAM, UPMC, University Paris VI, Paris, 14-19 (2006)

\section{Conclusion}

Applying active control to a vibrating system modifies the parameters of its movement. This technique has 